\title{
Is Utilizing a Glucometer in the Dental Office Effective in Improving Diabetic Patients' Oral Health?
}

\section{$\underline{\text { Original Article }}$}

Hengameh Khosropanah ${ }^{1}$, Nazila Lashkarizadeh ${ }^{2}$, Saman Mohammadzadeh ${ }^{3}$, Mohammadreza Mosallanezhad ${ }^{4}$

\author{
${ }^{1}$ Associate Professor of Periodontics, Department of \\ Periodontics, School of Dentistry, Shiraz University \\ of Medical Sciences, Shiraz, Iran \\ ${ }^{2}$ Resident of Periodontics, Department of Periodon- \\ tics, School of Dentistry, Shiraz University of Medi- \\ cal Sciences, Shiraz, Iran \\ ${ }^{3}$ Resident of Periodontics, Department of Periodon- \\ tics, School of Dentistry, Shiraz University of Medi- \\ cal Sciences, Shiraz, Iran \\ ${ }^{4}$ Dentist, Private Office, Shiraz, Iran.
}

Received:Jun 13, 2015

Accepted: Aug 11, 2015

\section{Corresponding Author: \\ Nazila Lashkarizadeh \\ Address:}

Resident of Periodontics, Department of Periodontics, School of Dentistry, Shiraz University of Medical Sciences, Shiraz, Iran.

E-mail: nazilalashkarizadeh@yahoo.com

Telephone: +989381285862

\begin{abstract}
Introdouction:

Periodontal treatment could have a positive effect on diabetes control. In this study, the researchers evaluated the efficacy of utilizing glucometer devices to detect glycemic levels in dental offices on inducing motivation towards the oral health care of diabetic patients.

Materials and methods: Eighty volunteer patients with moderate periodontitis were selected for participation in one of the two groups ( 40 diabetic patients and 40 non-diabetics); the two groups were unified based on age and sex. Each participant's baseline blood glucose level with 2 hours post-prandial test ( $2 \mathrm{hpp}$ ) and periodontal parameters of CAL, PI, and $\mathrm{BI}$ were measured, and the two groups underwent non-surgical periodontal treatment accompanied by comprehensive oral hygiene instructions. Then, 2 hpp was measured using a glucometer after 1 week and 1 month. All periodontal parameters were evaluated again 1 month after non-surgical treatment.
\end{abstract}

Results: The pattern of changes in $2 \mathrm{hpp}$ mean values over time was not similar for the two groups. In the diabetic patients, the mean of 2 hpp values after 1 week (T1) and 1 month (T2) significantly decreased when compared with that at baseline (TO) (both $p<0.001$ ). Moreover, the mean of $2 \mathrm{hpp}$ values at T2 was significantly lower than that of T1 $(p<0.001)$. All periodontal parameters significantly decreased at $\mathrm{T} 2 \mathrm{com}$ pared to TO in both groups, but the difference between two time points was significantly higher only for CAL in the diabetic group compared to the non-diabetic group $(p=0.022)$.

Conclusion:The use of a glucometer, as a proactive behavior to increase motivation, was not as effective as oral hygiene instructions on plaque control improvement in diabetic patients with chronic periodontitis.

Key words:

- Blood Glucose, analysis -Diabetes Mellitus -Oral Health 


\section{Introduction}

Diabetes mellitus (DM) is a chronic disease that is characterized by impaired glucose metabolism. Diabetes and its complications have been a major public health problem for many years. ${ }^{(1,2)}$ The prevalence of diabetes, according to $\mathrm{WHO}$, was approximately $10 \%$ in 2008 and is estimated to double by $2030 .{ }^{(2)}$ There are different types of diabetes: type 1 , type 2 , and gestational diabetes. Among these, type 2 diabetes, called non-insulin dependent or adult onset diabetes, is known to be the most common type $(>80 \%) .^{(3)}$

Diabetic patients are known to be susceptible to developing periodontitis. Periodontitis is a chronic inflammatory disease that is a response to the presence of subgingival biofilm; it leads to the destruction of supporting periodontal structures and eventually to tooth loss. ${ }^{(4)}$ Chronic periodontitis is associated with elevated levels of various systemic markers of inflammation, such as C-reactive protein (CRP) and interleukin 6 (IL-6). ${ }^{(3)}$ Periodontitis treatment modulates the related excessive systemic and local inflammatory events.

Chronic hyperglycemia, through the excessive accumulation of advanced glycation end products (AGEs), impairs vascular integrity and the normal synthesis, maturation, and maintenance of collagen. ${ }^{(5)}$ It also inhibits the proliferation and differentiation of PDL cells ${ }^{(6)}$ and impedes cellular migration and repair.(7) On the other hand, diabetes is associated with elevated levels of systemic markers of inflammation. ${ }^{(8)}$ For example, while studying diabetes type II, scholars noticed that both hemoglobin A1c (HbAlc) $>8 \%$ and random glucose levels were associated with higher levels of interleukin 1 beta (IL-1 $\beta$ ) in gingival crevicular fluid (GCF). ${ }^{(9)}$ In addition, in diabetes type I, the levels of prostaglandin E2 (PGE2) and IL-1 $\beta$ in GCF were elevated in both gingivitis and periodontitis. ${ }^{(10)}$

Recently there has been an emphasis on a twoway relationship between diabetes and periodontitis. ${ }^{(1,4)}$ Not only is diabetes considered a major risk factor for periodontitis, diabetic patients, compared to non-diabetics (depending on the level of their glycemic control), are 2 to 3 times more susceptible to developing periodontitis. Moreover, periodontal infection could negatively influence glycemic control and increase the prev- alence and severity of diabetes-related complications, including retinopathy, diabetic neuropathy, proteinuria, cardiovascular complications, and delayed wound healing. ${ }^{(1)}$ In previous studies, many intervention trials and randomized control trials (RCTs) have been conducted on the effect of periodontal treatment on the improvement of glycemic control in diabetic patients. ${ }^{(11,12)}$ Most findings have confirmed the positive influence of periodontal treatment on glycemic control. However, a study conducted by Auyeung et al., with one year of follow-up, obtained different results. ${ }^{(13)}$ Furthermore, several meta-analyses ${ }^{(14,}$ ${ }^{15)}$ on this subject confirmed that the treatment of periodontal disease in diabetic patients (at least in the short term) causes an average $0.4 \%$ reduction in their HbA1c levels. This value is equivalent to the effect of adding a second drug to a diabetes patient's pharmaceutical regimen and is clinically effective in reducing diabetic complications. ${ }^{(1)}$ A study conducted in 2010 stated $^{(16)}$ that diabetic patients' awareness of diabetes and its relationship to periodontal health is low and that most patients were not well-informed of the oral health complications of their disease and the requirements for proper preventive care. Hence, healthcare providers have recently focused on education and self-monitoring, through lifestyle and oral health programs, to manage diabetes and periodontitis in the long term. ${ }^{(1,17,18)}$ The logic behind oral health programs involves the impact of plaque control on eradicating inflammation, the initiator of a vicious circle of destructive periodontitis and diabetes. ${ }^{(1)}$ A study done by Kneckt et al. has demonstrated that diabetic patients who have been successful in managing their gingivitis have experienced better glycemic control and lower mean HbA1c levels. ${ }^{(19)}$ The present study aimed to find a way to motivate diabetic patients about plaque control. That is to say, we hypothesized that, if non-surgical periodontal treatment causes improvement in glycemic control among diabetic patients, then using a method in the clinic to compare their glycemic levels 1 week and 1 month after sub-gingival scaling would enable patients to objectively understand how periodontal care can help them control their diabetes and, hence, motivate them to pay more attention to plaque control.

This is crucial, because diabetic patients regard diabetes as a life-threatening disease, which is 
more worthwhile to care about than periodontitis. Therefore, we used a glucometer as an easy, economic, and accessible device to record glucose levels in the dental office.

\section{Materials and Methods}

The present study was designed as a prospective parallel study that compared groups of diabetics and healthy controls. All patients gave informed consent.

Due to the lack of an appropriate article comparing levels of 2-h post-prandial glucose ( $2 \mathrm{hpp})$ by using a glucometer after non-surgical periodontal treatment, the sample size was calculated based on the effect of intervention (non-surgical therapy) on diabetic patients. ${ }^{(11)}$ In this respect, the sample size needed, based on a comparison of the mean value of CAL in diabetic patients before $(3.14 \pm 1.08)$ and after $(2.8 \pm 1.09)$ intervention at $\alpha=0.05$ and power $=80 \%$, was determined to be 18 ; however, to increase reliability and comparability with the control group, the required sample size in both the case and control groups was set at 40 subjects.

80 volunteer patients (diabetic and non-diabetics) were selected from patients referred to the periodontal department from different dental centers. The inclusion criteria were as follows: In the case group, subjects diagnosed with diabetes mellitus type 2 were treated with an oral agent (metformin) according to the patient's medical history, aged over 18 years old, no antibiotic treatment during last 3 months, more than $30 \%$ of teeth affected by chronic periodontitis, no history of periodontal treatment, and the presence of at least 20 teeth. In the control group, no history or signs of diabetes were present in either the patients or their families; otherwise, all other criteria were the same as the case group. Subjects were cooperative and signed the consent forms. The participants, all of whom were referred from different dental centers, were divided into two groups as follows: a diabetic group of 40 patients (under oral hypoglycemic agent therapy) who, based on the default glucometer level (Easygluco Auto Coding ${ }^{\mathrm{TM}}$, Infopia Co., Korea), had a 2 hpp glycemic level $>140$ and a control group of 40 non-diabetic patients. Before the beginning of the study, each participant's age, weight, and height were documented to record his or her body mass index (BMI). Then, the fol- lowing measurements were performed: first, the plaque index (PI) (O'Leary) was measured. It is worth mentioning that, before the measurement, the patient's mouth was washed with water tape and then the patient chewed a disclosing tablet for at least $1 \mathrm{~min}$. Thereafter, the bleeding index (BI) (i.e., the Silness-Löe index) was prepared: a periodontal probe was introduced into the interproximal areas and, after 15 to $30 \mathrm{~s}$, bleeding was recorded when it was present. Then, using a William's probe, probing depth, mucogingival junction, and recession were measured on the following sites: 3 points buccally (mesio-buccal, middle root, and disto-buccal) and 3 points lingually (mesio-lingual, middle root, and disto-lingual) around all of the patient's teeth. The clinical attachment level (CAL) was calculated by adding recession to the probing depth measurements. Oral hygiene was instructed using a soft toothbrush (Oral B sensitive toothbrush) and a modified Bass technique on both the study model and the patient's mouth and was checked several times using the same toothpaste (Parodontax) and dental floss. Since the measured indices of the study were all quantitative, the intraclass correlation coefficient (rICC) was used to evaluate agreement. The rICC value for all parameters were greater than 0.85 , showing an acceptable level of agreement. All measurements were done by one dentist in separate chartings at baseline and 1 month after non-surgical treatment. Then, they were checked by an experienced periodontist.

During the study, all patients were advised to eat breakfast, including only a palm-sized piece of bread and a piece of white cheese about the size of a cube (15 gr). At least $2 \mathrm{~h}$ after having breakfast, the patients' blood glucose was tested with a glucometer (Easygluco Auto Coding ${ }^{\mathrm{TM}}$ ) in the dental office and documented. The periodontal therapy procedures were started with supragingival and subgingival scaling and root planing using cavitron (Joya) and Bracy curettes (HuFriedy) within 2 to 4 sessions, depending on the amount of the calculus and patient cooperation. One week later, again approximately $2 \mathrm{~h}$ after having breakfast, each patient's blood glucose was tested with a glucometer. One month later, during a recall session, all the above measurements and tests were performed and documented 
once again. Finally, the differences between blood glucose level, plaque, and bleeding index and the mean attachment level were measured.

\section{Statistical Analysis:}

Student's t-test was used to evaluate the continuous variables, such as height, weight, and age, and the differences between the mean attachment level, plaque, and bleeding indices found in the two groups. The chi-squared test was used to compare sex ratio and gender-based differences between the two groups. Blood glucose levels at different times were analyzed by repeated measures ANOVA. In this analysis, time of evaluation was considered the within-subject effect and the study groups the between-subject effect. Normality of variable distribution was evaluated with a Kolmogorov-Smirnov test. The significant level was set at 0.05 . The statistical analysis was performed using SPSS 16 software.

\section{Results}

In this study, 80 patients (40 diabetic and 40 non-diabetic patients) were evaluated. There were no significant differences in the baseline characteristics between the two groups. In the diabetic group,

there were 22 females and 18 males, while the control group consisted of 18 females and 22 males $(p=0.371)$. The mean age in the diabetic and the non-diabetic group was

$44.98 \pm 15.04$ and $39.5 \pm 14.98(p=0.107)$, respectively, and the mean BMI value was 26.73 \pm 3.7 and $27.52 \pm 5.24(\mathrm{p}=0.437)$, respectively. The blood glucose levels of all patients were evaluated before scaling and root planing, as well as 1 week and 1 month after the procedure was performed (Table 1).

There was a significant interaction effect between group and time $(p<0.001)$;

Table 1. Mean and standard deviation of blood glucose in diabetic and non-diabetic groups before, 1 week, and I month after scaling and root planing

\begin{tabular}{|c|c|c|c|c|}
\hline \multirow{2}{*}{ Parameter } & \multicolumn{3}{|c|}{ Time } & \multirow{2}{*}{ P-value } \\
\hline & Before (TO) & 1 week (T1) & 1 month (T2) & \\
\hline $\begin{array}{l}\text { Blood Glucose } \\
\text { (diabetic) }\end{array}$ & $216.15 \pm 56.58^{\mathrm{a}}$ & $193.60 \pm 45.95^{\mathrm{s}}$ & $186.40 \pm 44.02^{x}$ & $<0.001$ \\
\hline $\begin{array}{l}\text { Blood Glucose } \\
\text { (non-diabetic) }\end{array}$ & $83.38 \pm 13.67^{\mathrm{a}}$ & $78.30 \pm 12.90^{\mathrm{a}}$ & $80.38 \pm 11.01^{\mathrm{a}}$ & 0.134 \\
\hline
\end{tabular}

In each group, mean $2 \mathrm{hpp}$ values with the same lowercase letters were not statistically different. that is, the pattern of changes in 2 hpp mean value over time points was not similar for the two groups. More specifically, for DM patients, the mean of their $2 \mathrm{hpp}$ values at T1 $(193.60 \pm 45.95)$ and T2 (186.40 \pm 44.02$)$ significantly decreased when compared with that at T0 $(216.15 \pm 56.58)$ (both $\mathrm{p}<0.001$ ). Moreover, the mean of DM patients' 2 hpp values at T2 was also significantly lower than that at T1 $(\mathrm{p}<0.001)$. However, there was no significant difference between the means of the control group's $2 \mathrm{hpp}$ values at any of the three time points (all were $\mathrm{p}>0.05$ ).

The mean clinical attachment level (CAL), plaque, and bleeding indices (PI, BI) were assessed in the two groups before and 1 month after scaling and root planing (Table 2).

As shown in Table 2, the means of CAL, BI, and PI decreased 1 month after scaling and root planing, exhibiting statistically significant differences between those parameters before and after scaling and root planing in both groups $(\mathrm{p}<0.05)$.

Whereas the mean and standard deviation of differences in the mean CAL from $\mathrm{T} 0$ to $\mathrm{T} 2$ in diabetic patients was $-0.576 \pm 0.406$, in non-diabetic patients, they were $-0.364 \pm 0.411$, and these differences were statistically significant betweenthe two groups $(p=0.022)$.

The difference in BI from $\mathrm{T} 0$ to $\mathrm{T} 2$ in diabetic patients was $-0.116 \pm 0.199$ and $-0.097 \pm 0.0249$ in non-diabetic subjects; these differences were statistically not significant between the two groups $(p=0.542)$. Also, the mean and standard deviation of differences in PI at T0 to T2 in the two groups was as follows: $-0.09 \pm 0.05$ in the diabetic group and $-0.078 \pm 0.059$ in the non-diabetic group. However, these differences were not statistically significant ( $p=0.321$ ).

\footnotetext{
In each group, mean 2 hpp values with the same lowercase letters were not statistically different.
} 
Table 2. Distribution of mean clinical attachment level, bleeding index, and plaque index in diabetic (group 1) and non-diabetic (group 2) before and after scaling and root planing

\begin{tabular}{cccccc}
\hline \multirow{2}{*}{ Parameter } & Group & Before (T0) & $\begin{array}{c}\text { Time } \\
\text { After(T2) }\end{array}$ & Difference & P-value \\
& 1 & $2.97 \pm 0.63$ & $2.39 \pm 0.77$ & $-0.576 \pm 0.406$ & $<0.001$ \\
CAL & 2 & $2.86 \pm 0.88$ & $2.50 \pm 0.92$ & $-0.364 \pm 0.411$ & $<0.001$ \\
& P-value & 0.522 & & 0.022 & $<0.001$ \\
& 1 & $0.7315 \pm 0.1379$ & $0.6153 \pm 0.1472$ & $-0.1163 \pm 0.1988$ & 0.019 \\
BI & 2 & $0.649 \pm 0.164$ & $0.5521 \pm 0.1765$ & $-0.0969 \pm 0.0249$ & $<.001$ \\
& P-value & 0.017 & & $-0.0904 \pm 0.0502$ & $<0.001$ \\
& 1 & $0.6819 \pm 0.1324$ & $0.5919 \pm 0.1275$ & $-0.0782 \pm 0.0587$ & $<0.001$ \\
\hline
\end{tabular}

\section{Discussion}

Although, in the present study, the importance of periodontal treatment in decreasing diabetic patients' blood glucose level was emphasized to the diabetic patients in the dental office, this failed to motivate them to better manage plaque control. Both groups, diabetic and non-diabetic, showed similarly positive reactions after receiving mere education at baseline. It is worth mentioning that since this study, to the best knowledge of the researchers, is the first one to be conducted on the effect of informing and showing diabetic patients the importance of periodontal care on controlling blood glucose level, it suffers some limitations. Previous studies that aimed to improve oral hygiene in diabetic patients were planned according to the guidelines of different education programs and a glucometer has been used by only a few dentists exclusively for diabetes screening. For example, in two studies on the management of diabetic patients by dentists, Forbes et al Kunzel et al. ${ }^{(20)}$ and Forbes et al. ${ }^{(21)}$ concluded that, although dentists found it useful to give information to diabetic patients about the effect of oral hygiene on controlling diabetes, most of them merely participated in the discussion phase with patients and never exhibited a tendency to take proactive measures, such as using a finger-stick (glucometer) to measure blood glucose levels in their dental offices. Periodontists exhibited a greater tendency to involve themselves in proactive behaviors, but general dentists rarely assumed responsibility in this regard. ${ }^{(22)}$ Therefore, the researchers declare that, as the number of patients with diabetes increases, so does the responsibility of dentists to play an active role in helping their patients manage diabetes.

Saengtipbovorn et al. ${ }^{(23)}$, in their study, published in 2015, proposed a program in which lifestyle change and dental care, with 6 months of follow-up, could improve oral and systemic health in diabetic patients. The dental care program consisted of group and individual education on DM complications and their relationship to oral health, plus using a toothbrush, dental floss, and interproximal brush. Furthermore, monthly educational booster programs were performed. The success of this 6-month program in improving periodontal $(\mathrm{BI}, \mathrm{CAL}$, bleeding on probing (BOP)) and diabetic status (HbAlc and fasting plasma glucose (FPG)) seemed to the result of engaging dentists with individual patients and having educational booster sessions.

In the present study, the follow-up period was 1 month because the CALs were in the range of 2-3 $\mathrm{mm}$ and Morrison and Lowenguth demonstrated that, after 1 month of non-surgical periodontal treatment, the inflammation caused by periodontitis would ameliorate. ${ }^{(24)}$ Furthermore, the effect of non-surgical treatment on the decline of glycemic levels in diabetics is associated with the reduction of local and systemic inflammation related to periodontal infection. ${ }^{(1-3)} \mathrm{How}-$ ever, it seems that improvement in the glycemic levels of patients, as measured with a glucometer in a dental office, is advantageous in motivating diabetics to improve their periodontal care only while treating periodontal disease and resolving inflammation.

On the one hand, the present research investigated the influence of demonstrating the use of a 
glucometer in the dental office on the improvement of diabetic patients' plaque index. However, obtaining an optimal plaque index score appears to be dependent upon developing patients' dexterity at proper plaque removal. Thus, we should not overlook that this method could have a positive influence on diabetic patients when the dental practitioner engages him/herself in their disease management and that the use of a glucometer could be an easy and useful method to provide an overview of patients' glucose levels, thus enabling patients and dentists to confirm the positive effect of periodontal treatment on diabetes. Hence, in future studies, it might be worthwhile to consider the influence of this method on increasing diabetic patients' participation in long-term periodontal care follow-ups. On the other hand, this method seems to suffer some shortcomings, the first one being the short duration of its effects.

A review based on meta-analysis has reported that the effect of periodontal treatment on glycemic level reduction can be documented in a short time (3 months) ${ }^{(25,26)}$; therefore, if it is to be used merely as an aid for providing objective understanding to diabetic patients, it should be accompanied by proper education about the chronic features of periodontal disease that needs longterm maintenance.

Another point in the present study is using a glucometer as an easy-to-access device to measure chairside 2 hpp glycemic level in the dental office. As mentioned previously, finger-sticks have been used in the dental office in previous studies (20) as a device for diabetes screening, but most dentists were unwilling to use such devices to monitor glucose levels. Thus, it is crucial to inform dentists about the beneficial effects of glucometers.

Glucometers are accepted among diabetic patients as s self-monitoring devices for information about changes in glycemic level at short intervals. Moreover, they are accepted to be valuable in the primary health care setting in controlling diabetes. ${ }^{27,28)}$ A study has been conducted on the accuracy of glucometer measurements. ${ }^{(29)}$ A study carried out by Robel et al. ${ }^{(30)}$ maintains that the accuracy of glucometers depends upon many factors, such as the proper use of the devices, sample source, and patient parameters, including $\mathrm{pH}$, blood oxygen, hematocrit, whether the patient is taking vasopressor drugs, and changes in microcirculation. In addition, glucometers fail to be accurate when glucose values approach the endpoints of hypoglycemia and hyperglycemia. However, in euglycemia, the measurements are close to the real values. In the present study, glucometer measurements were utilized just to compare glucose levels at baseline, 1 week, and 1 month after periodontal treatment, not to make serious clinical decisions for diabetes; therefore, the application of glucometers in this study seems to be acceptable.

In contrast to most of the previous studies, which used markers, such as fasting blood sugar (FBS) and $\mathrm{HbAlc}$, to evaluate the level of glycemic control, we used $2 \mathrm{hpp}$. HbAlc is a reliable marker for detecting long-term diabetes control (during the last 3 months), while $2 \mathrm{hpp}$ is an appropriate marker for evaluating the level of diabetes control in short-term intervals and, hence, is suitable to be used in a dental office. $2 \mathrm{hpp}$ is also more helpful in giving information about diabetic complications, especially macro-vascular complications, than other markers, such as FBS. (27)

Based on the literature, the risk of periodontal disease in diabetic patients is influenced by demographic characteristics, glycol-hemoglobin level, and BMI ${ }^{(31)}$, while the clinical response to non-surgical periodontal therapy is influenced by baseline disease severity and BMI. ${ }^{(32)}$ Therefore, to exclude confounding factors in this study, the case and control groups were matched based on age, sex, disease severity, and BMI.

Among the periodontal parameters (BOP, PI, CAL), checked in the present study, only CAL appeared to improve statistically significantly after non-surgical treatment in diabetic patients compared to non-diabetics. This probably could not be explained by improved gingival health in diabetes patients, since well-controlled diabetic patients might respond to non-surgical treatment the same as healthy individuals, but uncontrolled diabetes might interfere with the normal healing process. ${ }^{(6)}$ Instead, it is likely that this result is associated with the higher inflammatory status of diabetic patients with periodontitis, which leads to technical errors while measuring CAL with a periodontal probe, as well as more significant differences in periodontal tissue after subgingival debridement and resolving the underlying 
inflammation.Based on this study, since internal medicine specialists are often the first members of a medical team to encounter diabetic patients, they are recommended to educate their patients on the importance of oral health, along with regular follow-ups in their office; they are expected to refer every diabetic patient to a dentist as well. Dental practitioners should also participate in an oral health education program that emphasizes lifestyle changes in diabetic patients.

\section{Conclusion}

Non-surgical periodontal treatment is effective in improving glycemic levels in diabetic patients; in addition, using a glucometer is an easy and useful method to obtain an overview of pa- tients' glucose level so that patients and dentists can confirm the positive impact of periodontal treatment on diabetes. However, in comparison to proper oral hygiene instruction, no additional benefits were derived from this method in terms of improving diabetic patients' plaque index.

\section{Acknowledgement}

The authors thank the Vice-Chancellory of Shiraz University of Medical Science for supporting this research. The authors also thank Dr. Vosugh of the Dental Research Development Center, of the School of Dentistry for the statistical analysis and Dr. Ehya Amal Saleh for .improving the use of English in the manuscript

\section{Refrences}

1. Preshaw P, Alba A, Herrera D, et al. Periodontitis and diabetes: a two-way relationship. Diabetologia 2012;55(1):21-31. doi: 10.1007/s00125-011-2342-y. Epub 2011.

2. Engebretson S, Kocher T. Evidence that periodontal treatment improves diabetes outcomes: a systematic review and meta-analysis. J Periodontol 2013;84(4 Suppl):S153-69. doi: 10.1902/jop.2013.1340017.

3. Mohan M, Jhingran R, Bains VK, et al . Impact of scaling and root planing on C-reactive protein levels in gingival crevicular fluid and serum in chronic periodontitis patients with or without diabetes mellitus. J Periodontal Implant Sci 2014;44(4):158-68. doi: 10.5051/jpis.2014.44.4.158. Epub 2014; 28.

4. Bascones-Martinez A, Matesanz-Perez P, Escribano-Bermejo M, et al. Periodontal disease and diabetes-review of the literature. Med Oral Patol Oral Cir Buca 2011; 16(6):e722-9.

5. Cortizo AM, Lettieri MG, Barrio DA, et al. Advanced glycation end-products (AGEs) induce concerted changes in the osteoblastic expression of their receptor RAGE and in the activation of extracellular signal-regulated kinases (ERK). Mol Cell Biochem 2003;250(1-2):1-10.2003;250(1-2):1-10.

6. Chang PC, Chung MC, Wang YP, et al. Patterns of diabetic periodontal wound repair: a study using micro-computed tomography and immunohistochemistry. J Periodontol 2012;83(5):644-52.

7. Grossi SG, Zambon JJ, Ho AW, et al. Assessment of risk for periodontal disease. I. Risk indicators for attachment loss. J Periodontol 1994;65(3):260-7.

8. Dandona P, Aljada A, Bandyopadhyay A. Inflammation: the link between insulin resistance, obesity and diabetes. Trends Immunol 2004;25(1):4-7.

9. Engebretson SP, Hey-Hadavi J, Ehrhardt FJ, et al. Gingival crevicular fluid levels of interleukin-1 $\beta$ and glycemic control in patients with chronic periodontitis and type 2 diabetes. J Periodontol 2004;75(9):1203-8.

10. Salvi GE, Yalda B, Collins JG, et al. Inflammatory mediator response as a potential risk marker for periodontal diseases in insulin-dependent diabetes mellitus patients. J Periodontol1997; 68(2):127-35.

11. Moeintaghavi A, Arab H, Bozorgnia Y, et al. Non-surgical periodontal therapy affects metabolic control in diabetics: a randomized controlled clinical trial. Aust Dent J 2012;57(1):31-7. doi: 10.1111/j.1834-7819.2011.01652.x.

12. Koromantzos PA, Makrilakis K, Dereka X, et al. A randomized, controlled trial on the effect of non-surgical periodontal therapy in patients with type 2 diabetes. Part I: effect on periodontal status and glycaemic control. J Clin Periodontol 2011 Feb;38(2):142-7. doi: 10.1111/j.1600-051X.2010.01652.x. Epub 2010; 29.

13. Auyeung L, Wang P-W, Lin R-T, et al. Evaluation of periodontal status and effectiveness of non-surgical treatment in patients with type 2 diabetes mellitus in Taiwan for a 1-year period. J Periodontol 2012;83(5):621-8.

14. Janket S-J, Wightman A, Baird A, et al. Does periodontal treatment improve glycemic control in diabetic patients? A meta-analysis of intervention studies. J Dent Res 2005;84(12):1154-9.

15. Teeuw WJ, Gerdes VE, Loos BG. Effect of periodontal treatment on glycemic control of diabetic patients: a systematic review and meta-analysis. Diabetes Care 2010;33(2):421-7. doi: 10.2337/dc09-1378.

16. Al Habashneh R, Khader Y, Hammad MM, Almuradi M. Knowledge and awareness about diabetes and periodontal health among Jordanians. J Diabetes Complications 2010;24(6):409-14. doi: 10.1016/j.jdiacomp.2009.06.001. Epub 
2009;22.

17. Deakin T, Cade J, Williams R, Greenwood D. Structured patient education: the Diabetes X-PERT Programme makes a difference. Diabet Med 2006;23(9):944-54.

18. Gillett M, Dallosso H, Dixon S, et al. Delivering the diabetes education and self management for ongoing and newly diagnosed (DESMOND) programme for people with newly diagnosed type 2 diabetes: cost effectiveness analysis. BMJ 2008; 336(7642): 491-495.

19. Kneckt MC, Syrjälä AMH, Knuuttila ML. Attributions to dental and diabetes health outcomes. J Clin Periodontol 2000;27(3):205-11.

20. Forbes K, Thomson WM, Kunzel C, et al. Management of patients with diabetes by general dentists in New Zealand. J Periodonto 2008;79(8):1401-8. doi: 10.1902/jop.2008.070640 .

21. Kunzel C, Lalla E, Lamster IB. Management of the patient who smokes and the diabetic patient in the dental office. J Periodontol2006;77(3):331-40.

22. Kunzel C, Lalla E, Lamster I. Dentists' management of the diabetic patient: contrasting generalists and specialists. Am J Public Health 2007;97(4):725-30. Epub 2007 Feb 28.

23. Saengtipbovorn S, Taneepanichskul S. Effectiveness of lifestyle change plus dental care (LCDC) program in improving glycemic and periodontal status in aging diabetic patients: a cluster randomized controlled trial. J Periodontol 2015:1-12.

24. Lowenguth RA, Greenstein G. Clinical and microbiological response to nonsurgical mechanical periodontal therapy. Periodontol 2000. 1995;9(1):14-22.

25. Simpson TC, Needleman I, Wild SH, et al. Treatment of periodontal disease for glycaemic control in people with diabetes. Cochrane Database Syst Rev 2010;(5):CD004714. doi: 10.1002/14651858.CD004714.pub2.

26. Chapple IL1, Genco R; Working group 2 of joint EFP/AAP workshop. . Diabetes and periodontal diseases: consensus report of the Joint EFP/AAP workshop on periodontitis and systemic diseases J Clin Periodontol 2013;40 Suppl 14:S10612. doi: $10.1111 /$ jcpe. 12077 .

27. Parkin CG, Brooks N. Is postprandial glucose control important? Is it practical in primary care settings? Clin Diabetes2002;20(2):71-6.

28. Parkin CG, Davidson JA. Value of self-monitoring blood glucose pattern analysis in improving diabetes outcomes. J Diabetes Sci Technol 2009;3(3):500-8.

29. Boren SA, Clarke WL. Analytical and clinical performance of blood glucose monitors. J Diabetes Sci Technol 2010;4(1):84-97.

30. Rebel A, Rice MA, Fahy BG. The accuracy of point-of-care glucose measurements. J Diabetes Sci Technol 2012;6(2):396-411.

31. Garcia D, Tarima S, Okunseri C. Periodontitis and glycemic control in diabetes: NHANES 2009-2012. J Periodontol 2014:1-10.

32. Michalowicz BS, Hyman L, Hou W, et al. Factors associated with the clinical response to nonsurgical periodontal therapy in people with type 2 diabetes mellitus. J Am Dent Assoc 2014;145(12):1227-39. doi: 10.14219/jada.2014.92.. 